\title{
Trends in adult chlamydia and gonorrhoea prevalence, incidence and urethral discharge case reporting in Mongolia from 1995 to 2016 - estimates using the Spectrum-STI model
}

\author{
Jugderjav Badrakh, ${ }^{a}$ Setsen Zayasaikhan, a Davaalkham Jagdagsuren, ${ }^{a}$ Erdenetungalag Enkhbat, ${ }^{a}$ Narantuya

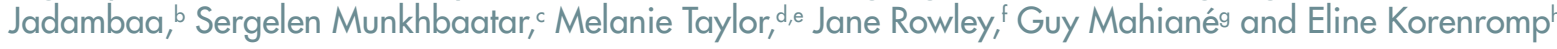 \\ Correspondence to Eline Korenromp (ekorenromp@avenirhealth.org)
}

Objective: To estimate Mongolia's prevalence and incidence trends of gonorrhoea and chlamydia in women and men 15-49 years old to inform control of STIs and HIV, a national health sector priority.

Methods: We applied the Spectrum-STI estimation model, fitting data from two national population surveys (2001 and 2008) and from routine gonorrhoea screening of pregnant women in antenatal care (1997 to 2016) adjusted for diagnostic test performance, male/female differences and missing high-risk populations. Prevalence and incidence estimates were then used to assess completeness of national case reporting.

Results: Gonorrhoea prevalence was estimated at 3.3\% (95\% confidence interval, 1.6-3.9\%) in women and $2.9 \%$ (1.6-4.1\%) in men in 2016; chlamydia prevalence levels were 19.5\% (17.3-21.9\%) and 15.6\% (10.0-21.2\%), respectively. Corresponding new incident cases in women and men in 2016 totalled 60334 (36 147 to 121 933) and 76893 (35 639 to 254 913) for gonorrhoea and 131306 (84 232 to 254316 ) and 148162 (71 885 to 462 588) for chlamydia. Gonorrhoea and chlamydia prevalence declined by an estimated 33\% and 11\%, respectively from 2001 to 2016. Comparing numbers of symptomatic and treated cases estimated by Spectrum with gonorrhoea case reports suggests that $15 \%$ of symptomatic treated gonorrhoea cases were reported in 2016 ; only a minority of chlamydia episodes were reported as male urethral discharge cases.

Discussion: Gonorrhoea and chlamydia prevalence are estimated to have declined in Mongolia during the early 2000s, possibly associated with syndromic management in primary care facilities and improving treatment coverage since 2001 and scale up of HIV/STI prevention interventions since 2003. However, prevalence remains high with most gonorrhoea and chlamydia cases not treated or recorded in the public health system.

$\mathrm{C}$ ontrol of sexually transmitted infections (STIs) and HIV is a health sector priority in Mongolia. Since 2001, syndromic case management is implemented in primary care facilities that lack capacity for laboratory diagnosis. Prevention services targeted at high-risk groups have been intensified since 2003 with support from the Global Fund to Fight AIDS, Tuberculosis and Malaria. ${ }^{1}$ In Mongolia, laboratory-diagnosed syphilis, gonorrhoea and trichomoniasis, as well as syndromically diagnosed male urethral discharge (UD) and genital ulcer disease from health facilities not doing laboratory diagnosis, are reportable; approximately 15000 new STI cases are registered annually. ${ }^{2-5}$ However, the true burden is believed to be higher due to undiagnosed and untreated cases, cases treated but not reported through private-sector facilities and those self-treated through pharmacies. $^{6-8}$

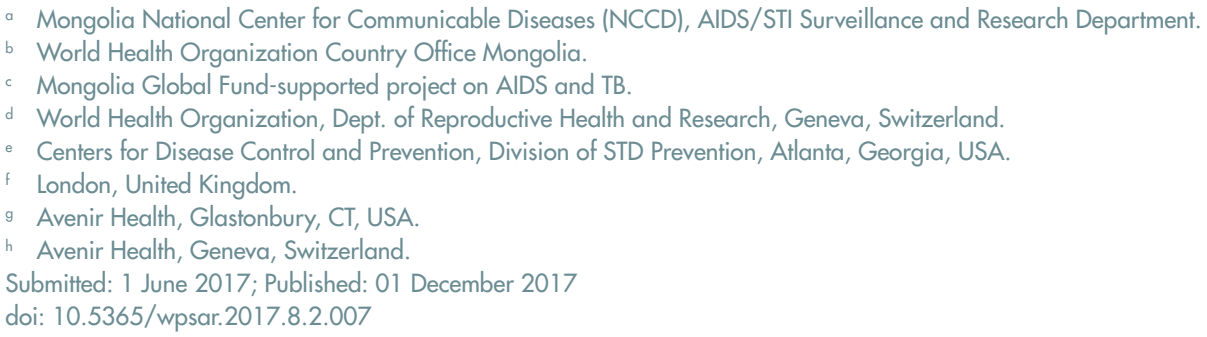


Syphilis surveillance draws on periodic serological surveys and routine, near-universal screening among pregnant women attending antenatal care (ANC). For gonorrhoea and chlamydia, however, no such systematic measurement is in place.

In 2017 Mongolia, with support from the World Health Organization (WHO) and Avenir Health, estimated its adult prevalence trends for chlamydia and gonorrhoea using the Spectrum-STI model ${ }^{9}$ to inform strategic planning for its STI response and strengthen its STI surveillance system.

The Spectrum-STI tool estimates trends in adult prevalence and incidence of STIs at the national level using data from routine STI surveillance and populationbased surveys. ${ }^{9}$

This article presents Spectrum estimates of adult prevalence and incidence of gonorrhoea and chlamydia in Mongolia from 1995 to 2016 using prevalence survey data. Estimated male gonorrhoea and chlamydia case numbers were compared to UD case reports to estimate treatment coverage and reporting completeness. This study represents the first national-level STI trend estimation in an Asian country using an internationally agreed approach and assumptions.

\section{METHODS}

\section{Overview}

The Spectrum-STI tool (http://avenirhealth.org/softwarespectrum.php $)^{9}$ estimated prevalence and incidence of gonorrhoea and chlamydia in adults aged 15-49 years. Data and assumptions were reviewed at a three-day technical workshop held in Mongolia in February 2017. Participants included representatives of the Ministry of Health, HIV/AIDS and Maternal and Child Health programmes, the central reference laboratory and partners supporting or implementing the national HIV/STI response. Mongolia-specific parameter values and results from the base-case analysis were agreed at the workshop and are summarized here. Spectrum default parameter values have been described elsewhere. ${ }^{9}$

\section{Prevalence estimation}

National prevalence levels for adult women were estimated over time as a moving average through all data points.

For both STIs, prevalence data were identified from studies conducted between 1995 and 2016 in representative general adult populations. For Mongolia, this included pregnant women attending ANC; no prevalence data were identified from any other low-risk populations.

Prevalence data from each study were adjusted for sensitivity and specificity of diagnostic tests used ${ }^{10-12}$ (Supplemental Digital Content (SDC) 1). For gonorrhoea the national data from routine screening of women attending ANC (SDC2) used culture or Gram stain on cervical or vaginal swabs; sensitivity of these tests was set at 35\% to reflect challenges in testing in routine care settings.

Each prevalence data point was adjusted upward by $10 \%$ to account for the contribution of higher-risk populations. ${ }^{10}$

National sample surveys were assigned a weight of $100 \%$ (the maximum, given that these should be nationally representative). Routine screening data were assigned a $40 \%$ weight, as agreed at the national workshop, as these were not nationwide or systematically sampled. Since the $40 \%$ value was somewhat arbitrary, we present estimates using different weights as sensitivity analysis.

Since no prevalence data were available for men, male prevalence was inferred from female estimates by applying a time-constant male-to-female prevalence ratio of 0.86 (range $0.58-1.15$ ) for gonorrhoea and 0.80 (range 0.53-1.07) for chlamydia with uncertainty bounds incorporating both uncertainty in female prevalence and in the male-to-female ratio. ${ }^{10}$

The 95\% uncertainty or confidence intervals were generated to account for binomial sampling variability in prevalence observed in the data and modelling error. ${ }^{9}$ Test-adjusted prevalence rates were simulated (in 10000 replications) following $\beta$ distributions to which we added random terms in the logit scale; the random terms were sampled from a uniform distribution on residuals obtained after fitting the original data set. 
Incidence estimation and STI episode durations

Incidence was estimated by dividing estimated prevalence by an assumed average duration of infection. ${ }^{13}$ The $95 \%$ confidence intervals on incidence reflect uncertainty in both the underlying prevalence (estimated by bootstrap) and in the duration of infection set at $\pm 50 \%$.

STI episode durations were as assumed in the WHO 2012 global and regional estimates. ${ }^{10}$ In the WHO estimates, the region that Mongolia is a member of was assumed to have intermediate treatment coverage. ${ }^{10} \mathrm{How}$ ever, following discussions at the national workshop, and lacking national population-based data about STI treatment coverage, we decided to use longer STI durations, reflecting low treatment coverage.

Assuming 35\% treatment coverage of symptomatic gonorrhoea and chlamydia episodes in men and 22.5\% in women, ${ }^{10,14}$ we calculated average durations of gonorrhoea and chlamydia episodes weighted between the fractions treated and untreated (SDC3). The average duration in men was 0.32 years for gonorrhoea and 0.86 years for chlamydia and 0.47 years for gonorrhoea and 1.22 years for chlamydia in women (SDC3).

\section{STI case reporting completeness}

An expected case load for UD was estimated from Spectrum-estimated case incidence, assuming that $64 \%$ of gonorrhoea cases and $14 \%$ of chlamydia cases are symptomatic and $35 \%$ of these are treated ${ }^{10}$ (SDC3). The Spectrum estimates of symptomatic gonorrhoea cases and UD cases were then compared to national-level case reports for laboratory-diagnosed gonorrhoea and UD (a non-overlapping set of cases without laboratory diagnosis) from 1995 to 2016 collected by the National Center for Communicable Diseases ${ }^{15,16}$ (SDC4) to estimate reporting completeness.

\section{Sensitivity analysis}

Univariate sensitivity analyses assessed the sensitivity of 2016 estimates to key assumptions and Mongolia-specific input data and assumptions: the weight of routine ANC screening data points; gonorrhoea relative to national ANC surveys; the sensitivity of culture and wet-mount in routine ANC gonorrhoea screening; the gonorrhoea prevalence data used; the decline rate in chlamydia (based on few data points) relative to that in gonorrhoea (based on a longer and more continuous time series); and (as determinant of reporting completeness) the gonorrhoea incidence rate in men 50-64 years. More general and global assumptions of the Spectrum methodology were addressed elsewhere. ${ }^{9}$

\section{RESULTS}

\section{Gonorrhoea and chlamydia prevalence}

Two national surveys were identified from the general population; both measured gonorrhoea and chlamydia in pregnant women attending ANC in $2001^{17}$ and $2008^{18}$ (SDC2, Fig. 1A \& B). For gonorrhoea, national prevalence data were also available for 1997 to 2016 from routine screening of women attending ANC.

For gonorrhoea in women, Spectrum estimated a stable $5.0 \%$ prevalence from 1995 to 2001 followed by a decline to $3.3 \%$ (95\% confidence interval, $1.6-3.9 \%$ ) in 2016 (Fig. 1A). For chlamydia, estimated prevalence fell from 2001 (the year of the first survey) and 2008 (the year of the second and final survey), and the model thereafter assumed stable prevalence. In 2000-2001 chlamydia prevalence was 21.9\% (19.5-24.4\%) and in 2016 19.6\% (17.3-21.9\%, Fig. 1B). From 2001 to 2016, gonorrhoea and chlamydia prevalence declined by $33 \%$ and $11 \%$, respectively.

No data were identified for men; therefore, male gonorrhoea and chlamydia estimates were based on female estimates (see Methods). In men, gonorrhoea prevalence was estimated at $4.3 \%$ between 1995 and 2001 and falling to $2.9 \%(1.6-4.1 \%)$ by 2016 . For chlamydia, prevalence was estimated at $17.5 \%(15.6-19.5 \%)$ in 2000 and $15.6 \%(10.0-21.2 \%)$ in 2016.

\section{Gonorrhoea and chlamydia incidence}

Fig. 1C \& D show estimated trends in gonorrhoea and chlamydia incidence. In 2016, Spectrum estimated 60334 (36 147-121 933) and 76893 (35 639 to 254 913) new gonorrhoea cases in women and men aged 15-49 years, respectively and 131306 (84 232 to $254316)$ new cases of chlamydia in women and 148162 (71 885 to 462588 ) in men (Table 1). For both STIs, incidence was higher in men than in women despite higher prevalence in women, reflecting longer average duration of both infections in women than in men. 
Fig. 1. Prevalence of (A) gonorrhoea and (B) chlamydia and incidence rate of (C) gonorrhoea and (D) chlamydia in women 15-49 years in Mongolia

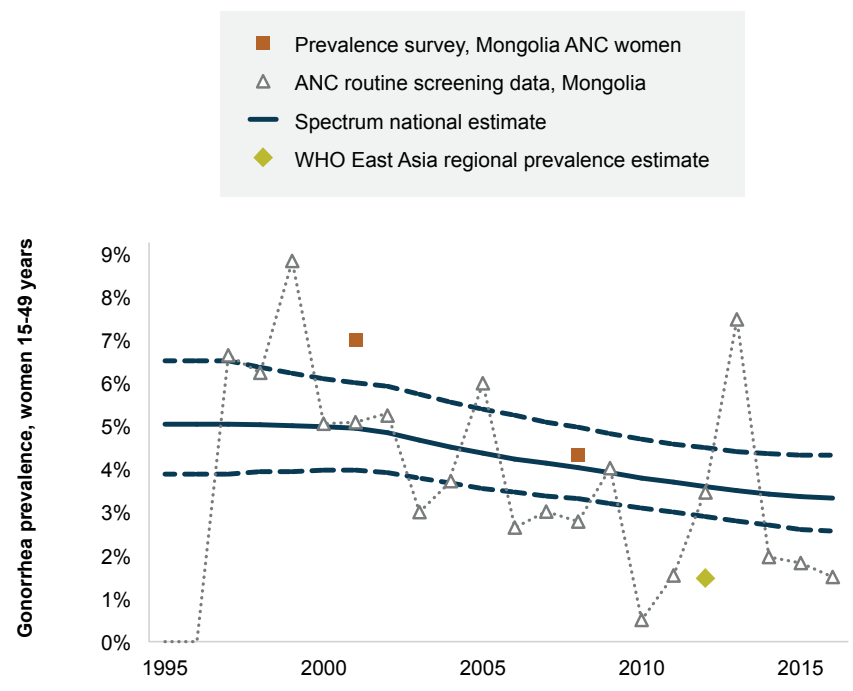

A

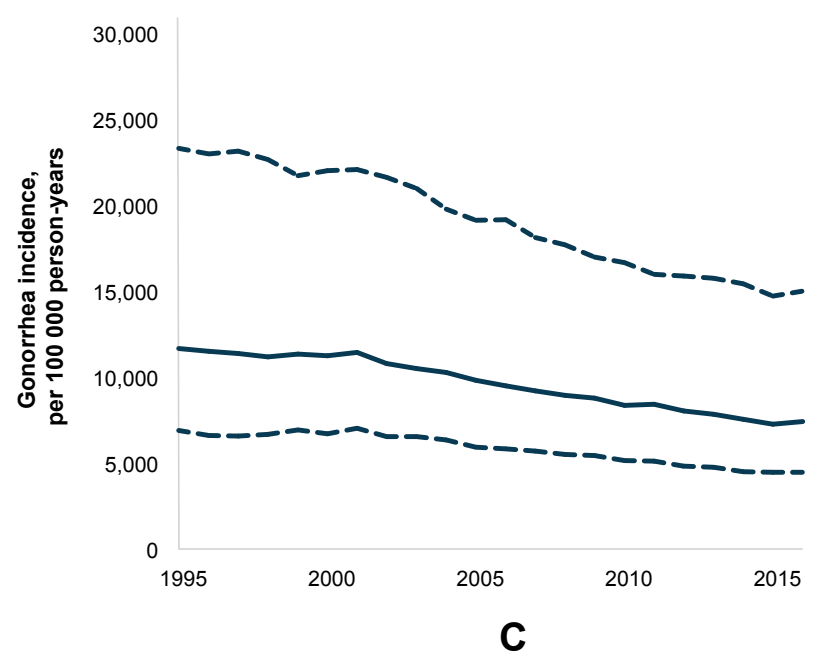

- Prevalence survey, Mongolia ANC women

- Spectrum national estimate

WHO East Asia regional prevalence estimate

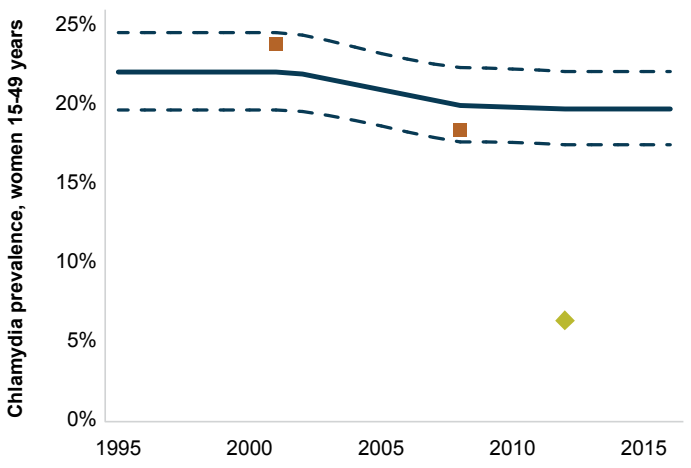

B

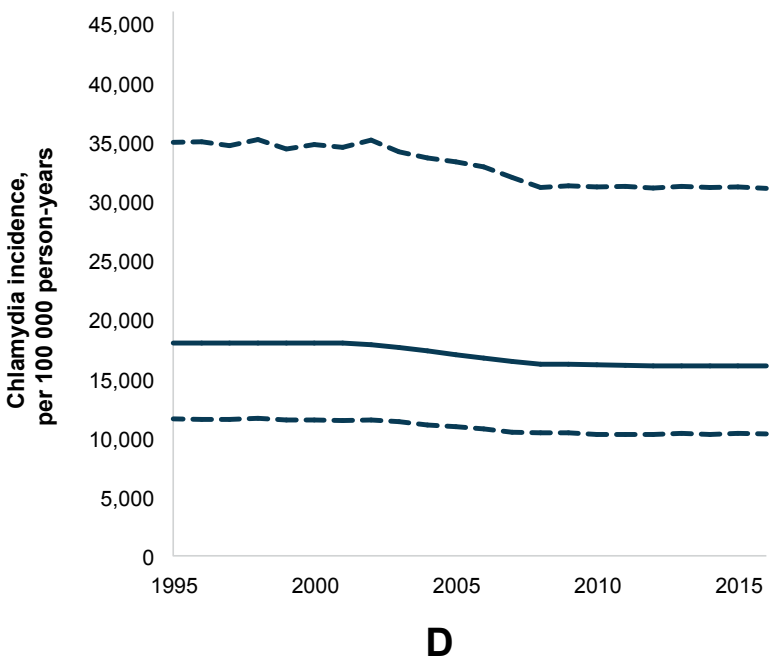

Prevalence data are shown after adjustments for diagnostic test performance (including routine ANC screening-based gonorrhoea prevalence levels adjusted for 35\% estimated sensitivity of culture and wet-mount, see SDC1), and missing high-risk populations (SDC2), as described in Methods. WHO regional prevalence estimates for East Asia in $2012^{10}$ are shown for comparison, but were not used in statistical estimation for Mongolia. Solid lines are best estimates: dotted lines are $95 \%$ Confidence Intervals. Data and prevalence estimates shown are for women $15-49$ years.

Gonorrhoea's estimated case incidence rate declined in women from 11 650/100 000 in 1995 to 7409 in 2016 and in men from 13991 to 9316 (Fig. 1C). Over this period, chlamydia incidence fell from 17953 to $16023 / 100000$ in women and from 20374 to 18 184/100 000 in men (Fig. 1D). For chlamydia, annual incident case numbers increased slightly from 1995 to 2002, a period when prevalence (Fig. 1B) and incidence rates (Fig. 1D) were estimated to have been stable; population growth implied slightly increasing annual case numbers (Fig. 2B). From 2002 to 2016, annual chlamydia cases were stable (Fig. 2B), reflecting the counterbalancing effects of declining prevalence and incidence rates (Fig. 1B and 1D) and population growth.

\section{Gonorrhoea reporting completeness and treat- ment coverage}

Fig. 2A shows Spectrum-estimated incident gonorrhoea cases in men from 1995 to 2016 split into episodes symptomatic and asymptomatic, treated and untreated and reported and unreported. Comparing national gonorrhoea case reports (SDC4) with Spectrum estimates of the number of men who were symptomatic and treated, 
Table 1. Spectrum-estimated prevalence and incidence rate (per 100000 person-years) of gonorrhoea and chlamydia in women and men 15-49 years, Mongolia in 2016

\begin{tabular}{llllll}
\hline STI & Metric & Women & \multicolumn{3}{l}{ Men } \\
\hline \multirow{3}{*}{ Gonorrhoea } & Point estimate & $95 \% \mathrm{Cl}$ & Point estimate & $95 \% \mathrm{Cl}$ \\
\cline { 2 - 6 } & Prevalence & $3.3 \%$ & $1.6-3.9 \%$ & $2.9 \%$ & $1.6-4.1 \%$ \\
& $\begin{array}{l}\text { Incidence rate per 100 000 total } \\
\text { adult population }\end{array}$ & 7409 & $4439-14974$ & 9316 & $4318-14,314$ \\
& New incident cases, 15-49 years & 60334 & $36147-121933$ & 76893 & $35639-254913$ \\
Chlamydia & Prevalence & $19.5 \%$ & $17.3-21.9 \%$ & $15.6 \%$ & $10.0-21.2 \%$ \\
& Incidence rate per 100 000 total & 16023 & $10279-31034$ & 18184 & $8823-27546$ \\
& adult population & & & & \\
& New incident cases, 15-49 years & 131306 & $84232-254316$ & 148162 & $71885-462588$ \\
\hline
\end{tabular}

$95 \% \mathrm{Cl}=95 \%$ confidence interval

Fig. 2. Spectrum-estimated incident gonorrhoea and chlamydia cases in men 15-49 years by treatment and reporting status, Mongolia
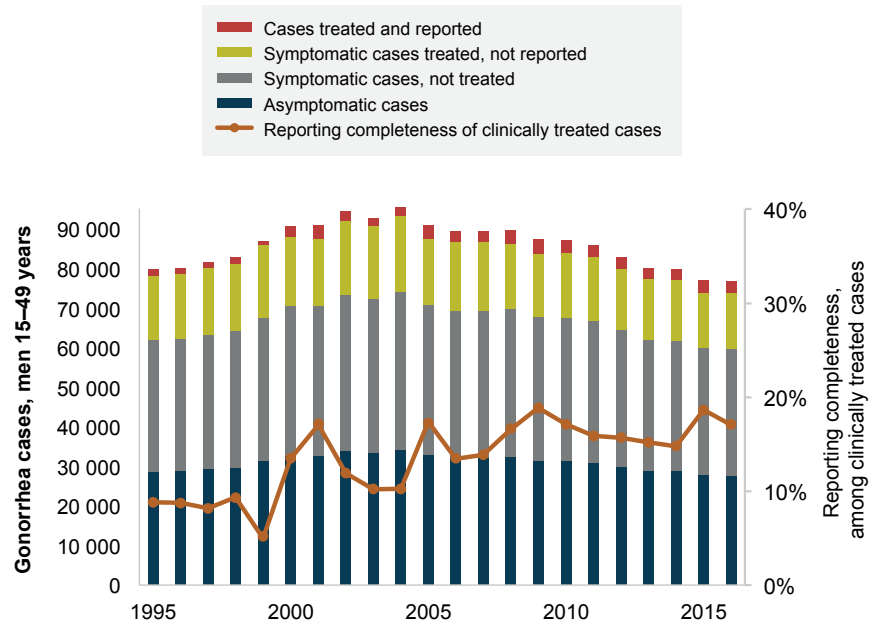

A
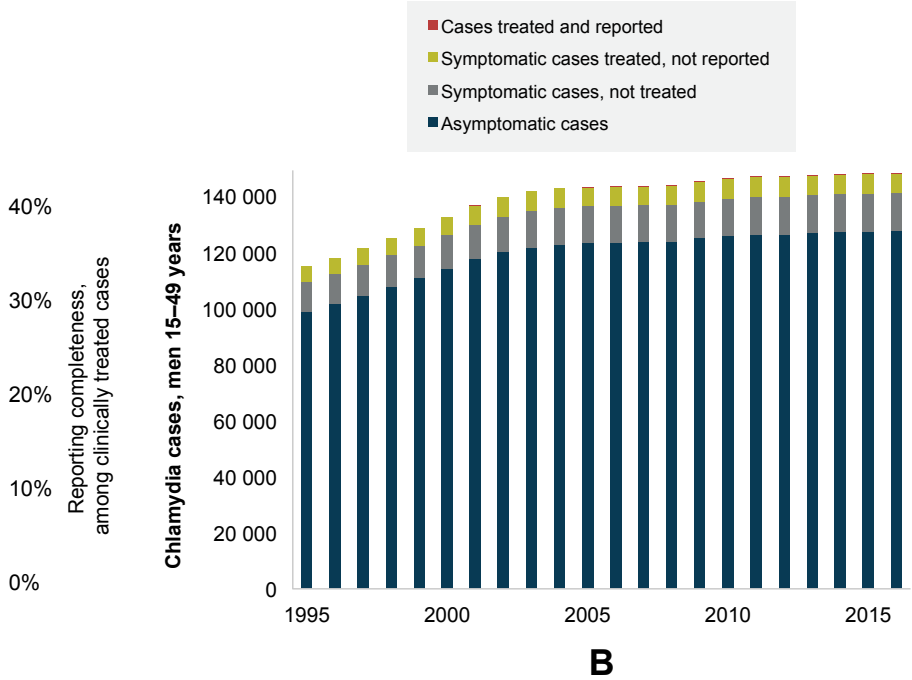

Reporting completeness is expressed as the reported gonorrhoea plus urethral discharge cases, relative to estimated incident gonorrhoea, chlamydia and other UD cases treated in clinics for which the calculation is detailed for year 2016 in Table 2.

reporting completeness for gonorrhoea cases treated in clinics improved from $8 \%$ in 1997 to $15 \%$ in 2016 (Table 2).

Chlamydia treatment and reporting coverage, 1995-2016

Of the Spectrum-estimated 148162 chlamydia cases in men aged 15-49 years in 2016, some 20743 were expected to be symptomatic of which 7260 would have been treated (Table 2 and Fig. 2B).

Laboratory diagnosis is not commonly practiced for chlamydia and chlamydia is not reportable; chlamydia cases in men should instead be reported as UD cases per the syndromic management policy. However, reported UD cases ranged between just 342 and 1648 in 2001 and in 2005 to 2015; years 2002 to 2004 were missing.

In 2016, of population-level estimated incident gonorrhoea plus chlamydia cases (76 893 gonorrhoea plus 148162 chlamydia; total 225 055), the reported 2625 gonorrhoea cases plus 606 UD cases covered only $1.4 \%$. This calculation assumed all UD cases were due to gonorrhoea and/or chlamydia; in reality, not all UD cases are caused by gonorrhoea or chlamydia (SDC5), thus these reporting completeness estimates are optimistic. Most unreported chlamydia cases were asymptomatic 
Table 2. Incident cases of gonorrhoea, chlamydia and urethral discharge and the subset who are treated and reported in men 15-49 years in Mongolia, 2016

\begin{tabular}{|c|c|c|c|}
\hline & Gonorrhoea & Chlamydia & Comment \& source \\
\hline New incident cases (Spectrum estimate) & 76893 & 148162 & Spectrum-STI estimate \\
\hline $\begin{array}{l}\text { Gonorrhoea or chlamydia cases developing UD/ } \\
\text { symptoms (Spectrum estimate) }\end{array}$ & 49212 & 20743 & $\begin{array}{l}\text { Assuming } 64 \% \text { of male gonorrhoea }{ }^{10} \text { and } \\
14 \% \text { of male chlamydia cases develop } \\
\text { symptoms }{ }^{13,19} \& \text { SDC3 }\end{array}$ \\
\hline $\begin{array}{l}\text { UD cases due to gonorrhoea or chlamydia } \\
\text { treated in a clinic (Spectrum estimate) }\end{array}$ & 17224 & 7260 & $\begin{array}{l}\text { Assuming } 35 \% \text { of gonorrhoeal \& chlamydial } \\
\text { UD cases seek and get treatment }{ }^{10} \& \text { SDC3 }\end{array}$ \\
\hline Actual case reports & \multicolumn{2}{|c|}{$\begin{array}{l}\text { Gonorrhoea: } 2625 \\
\text { UD (unknown etiology): } 606\end{array}$} & $\begin{array}{l}\text { Mongolia National Center for Communicable } \\
\text { Diseases }\end{array}$ \\
\hline $\begin{array}{l}\text { Gonorrhoea reporting completeness among } \\
\text { gonorrhoea cases treated in clinics }\end{array}$ & \multicolumn{2}{|c|}{$15 \%$} & $=2625 / 17224$ \\
\hline $\begin{array}{l}\text { Reported cases relative to population-level } \\
\text { incidence-gonorrhoea and chlamydia }\end{array}$ & \multicolumn{2}{|c|}{$1.4 \%$} & $=(2625+606) /(76893+148162)$ \\
\hline
\end{tabular}

$\mathrm{UD}=$ urethral discharge

(69\% in 2016, Fig. 2B), symptomatic but not treated (20\% at 2016) or treated but not reported (10\% in 2016).

\section{Sensitivity analysis}

Excluding routine ANC data from estimations, increased estimated gonorrhoea rates in 2016 (4.2\% instead of $2.9 \%$ in men) and reporting completeness of male gonorrhoea cases was correspondingly lower. Conversely, when ANC routine data points were given an increased weight, namely the same weight as each ANC survey, the estimated gonorrhoea prevalence and incidence fell slightly (male prevalence $2.7 \%$ instead of $2.9 \%$ ) and gonorrhoea reporting completeness increased.

Gonorrhoea results were also very responsive to the assumed sensitivity of culture in routine ANC-based screening: when varying the sensitivity between 25$75.7 \%{ }^{9}$ male prevalence in 2016 varied from $1.5-3.9 \%$ and gonorrhoea reporting completeness from $11-29 \%$.

Had gonorrhoea prevalence in Mongolian men in 2016 been as low as 1.28\% (the WHO 2012 estimate for men in East Asia) instead of our national estimate of $2.9 \%$, then gonorrhoea reporting completeness would have been $34 \%$ instead of $15 \%$ completeness.

Our analysis assumed that national case report was for men 15-49 years old. In fact, some reported cases will have been from older men. Estimated gonorrhoea reporting completeness would be $13 \%$ instead of the best estimate of $15 \%$ if we assume additional gonorrhoea and chlamydia cases to occur in men above 49 years at a rate equal to men $15-49$ years.

Finally, Spectrum estimates for chlamydia were based on two national surveys (2001 and 2008) and it was assumed that prevalence was constant after 2008. If chlamydia prevalence, however, fell between 2008 and 2016 at the same rate as gonorrhoea prevalence fell, the chlamydia prevalence in 2016 would have been 13\% rather than $15.6 \%$.

\section{DISCUSSION}

Prevalence trend estimations for Mongolia generated using the Spectrum-STI model and data from ANC-based surveys and routine screening indicate that prevalence of both chlamydia and gonorrhoea fell from 2001 to 2016. However, prevalence levels remained extremely high: for both STIs, Mongolia's estimated prevalence in 2012 was considerably above WHO estimates for the East Asia and central Asia regions ${ }^{10}$ (Fig. 1A \& B).

Chlamydia was estimated five times more prevalent than gonorrhoea as shown in the WHO 2012 estimates globally and for Asia. ${ }^{10}$ From 2001 to 2016, the estimated decline was stronger for gonorrhoea than for chlamydia, reflecting the gonorrhoea decline observed in ANC routine testing. We cannot exclude that for chlamydia the decline from 2001 to 2008 also continued after 2008 (see Table 3); however, there are no data 
Table 3. Sensitivity analysis - effect of varying (selected) assumptions and values on national estimates of gonorrhoea and chlamydia prevalence and incidence and the estimated reporting completeness for symptomatic treated gonorrhoea in Mongolian men 15-49 years, in 2016

\begin{tabular}{|c|c|c|c|c|c|c|c|c|}
\hline \multirow{2}{*}{ Parameter } & \multirow{2}{*}{$\begin{array}{l}\text { Default } \\
\text { assumption }\end{array}$} & \multicolumn{2}{|c|}{$\begin{array}{l}\text { Alternative } \\
\text { assumption }\end{array}$} & \multicolumn{2}{|c|}{ Prevalence } & \multicolumn{2}{|c|}{ Incidence per 100000} & \multirow{2}{*}{$\begin{array}{c}\text { Gonorrhea } \\
\text { case } \\
\text { reporting } \\
\text { completeness }\end{array}$} \\
\hline & & Lower & Upper & Gonorrhoea & Chlamydia & Gonorrhoea & Chlamydia & \\
\hline Best/default estimate & & & & $2.9 \%$ & $15.6 \%$ & 9316 & 18184 & $15.2 \%$ \\
\hline $\begin{array}{l}\text { Weight of routine ANC } \\
\text { screening data points, }\end{array}$ & $40 \%$ & $0 \%$ & & $4.2 \%$ & As default & 13735 & As default & $10 \%$ \\
\hline $\begin{array}{l}\text { gonorrhoea, relative to } \\
\text { national ANC surveys }\end{array}$ & & & $100 \%$ & $2.7 \%$ & As default & 8724 & As default & $16 \%$ \\
\hline \multirow{2}{*}{$\begin{array}{l}\text { Sensitivity of culture and } \\
\text { wet-mount, in routine ANC } \\
\text { gonorrhoea screening }\end{array}$} & $35 \%$ & $25 \%$ & & $3.9 \%$ & As default & 12622 & As default & $11 \%$ \\
\hline & & & $75.7 \%^{9}$ & $1.5 \%$ & As default & 4841 & As default & $29 \%$ \\
\hline $\begin{array}{l}\text { Gonorrhoea prevalence as } \\
\text { WHO's East Asia regional } \\
\text { estimate of } 2012^{10}\end{array}$ & $\begin{array}{l}\text { Fitted to } \\
\text { Mongolia } \\
\text { data }\end{array}$ & & & $1.28 \%$ & As default & 4172 & As default & $34 \%$ \\
\hline $\begin{array}{l}\text { Chlamydia prevalence: } \\
\text { decline from 2008-2016 } \\
\text { proportional to the } \\
\text { estimated prevalence } \\
\text { decline over 2008-2016 in } \\
\text { gonorrhoea }\end{array}$ & $\begin{array}{l}\text { Constant } \\
\text { from } \\
2008-2016\end{array}$ & & & As default & $13.0 \%$ & As default & 15138 & As default \\
\hline $\begin{array}{l}\text { Gonorrhoea incidence rate } \\
\text { in men } 50-64 \text { years }\end{array}$ & 0 & & $\begin{array}{l}\text { Same as } \\
\text { at } 15-49 \\
\text { years }\end{array}$ & As default & As default & As default & As default & $13 \%^{1}$ \\
\hline
\end{tabular}

post-2008 to establish this. Conversely, the estimated chlamydia decline from 2001 to 2008, based on two surveys, is our best estimate but is not as robust as a trend estimate based on multiple data points might have been. Estimates of chlamydia rates and especially their time trend are therefore less certain than for gonorrhoea.

The declines in gonorrhoea and chlamydia prevalence are attributable to several factors including the expanded HIV/STI response and scale-up of (Global Fund-supported) HIV/STI prevention interventions including outreach services with communication, counselling and HIV and STI testing for key groups since 2003. ${ }^{1}$ The declines are in line with Spectrum estimations for syphilis in ANC women. ${ }^{20}$

Declining prevalence of both gonorrhoea and chlamydia based on ANC data contrasts with stable or possibly increasing gonorrhoea and chlamydia prevalence in female sex workers (FSW) in Ulaanbaatar sampled through Integrated Bio-Behavioural Surveillance; FSW gonorrhoea prevalence increased from $13.6 \%$ in $2002^{21,22}$ to $15.6 \%$ in $2009^{23}$ and from $19.3 \%$ to $24.5 \%$ for chlamydia. This trend may be a true increase or reflect that in 2009 a higher-risk FSW population was sampled.
While gonorrhoea is the predominant cause of UD cases seen in clinics, ${ }^{15,16}$ at the population level the prevalence, incidence and case numbers are much higher for chlamydia. Still large numbers of gonorrhoea and especially chlamydia cases are not treated because many infections in both men and women do not cause symptoms and because over half of symptomatic chlamydiainfected adults do not access treatment.

Comparison of reported male gonorrhoea case numbers with Spectrum-estimated incident cases suggests that in 2016 15\% of male symptomatic gonorrhoea cases were treated and reported through public sector providers. Estimated reporting completeness improved after 2001 (8\%), coinciding with Mongolia's phased roll-out of syndromic STI treatment from a WHO-supported pilot in $2001^{1}$ to nationwide implementation in $2005 .^{24,25}$ In Spectrum simulations, treatment rates were assumed constant over time. If, however, treatment coverage improved then annual numbers of new cases (for a given prevalence) may have been higher and reporting completeness lower. The low reporting completeness highlights the need to strengthen Mongolia's national reporting system and ensure it covers both public and private providers. ${ }^{8}$ National surveys in 2010 and 2014 found that up to half 
of self-reported STI treatments were in the private sector (including purchases from pharmacies), ${ }^{14,26}$ yet only $9 \%$ of reported cases were from private clinics in $2015 .^{24}$

Despite recent declines, Mongolia has STI rates higher than neighbouring countries. This probably reflects persistent poor coverage of effective STI treatment by qualified providers. The high STI rates constitute a persistent risk factor for the possible future spread of $\mathrm{HIV}^{27}$

\section{Limitations}

The estimations are limited by the quality and quantity of prevalence data, modelling assumptions and assumptions made when data was lacking. ${ }^{9}$ For prevalence estimation, uncertain assumptions include that all prevalence data were from ANC women who may (as for HIV) not be representative for non-ANC non-pregnant women. ${ }^{28}$ Uncertainties particularly affected results for men (based on female estimates, applying a global fixed male-tofemale prevalence ratio) and results for chlamydia (with less national data than gonorrhoea).

Incidence estimates depended on treatment coverage and assumed durations of treated and untreated infections for which longitudinal data are lacking. Assumed proportions of episodes that become symptomatic were calibrated on WHO estimates for the East Asia region ${ }^{10}$ not on Mongolia-specific data. Proportions of symptomatic episodes that get treated were also taken from regional-level $\mathrm{WHO}$ assumptions ${ }^{10}$ where we situated Mongolia as a country with low treatment coverage, an assumption we could not validate against populationbased local data.

Finally, the assessment of gonorrhoea and UD case reporting completeness required additional assumptions, most of which were global rather than Mongolia-specific.

\section{Implications for surveillance and program-} matic response

The last national STI survey in Mongolia was conducted in 2008 in ANC women, and as of 2017 no survey has measured STI prevalence levels in low-risk men. Mongolia would benefit from regular population-based prevalence measurements looking at multiple STIs in low-risk men and women. These do not need to be large surveys but should be carefully designed to identify trends over time. Opportunities may be developed for tagging affordable sentinel STI screening onto existing data collection platforms. For example, screening and treatment for chlamydia in adolescents, as recommended in the WHO global STI strategy $2016-2021,{ }^{29}$ may yield useful data but is as yet not implemented in Mongolia. Reliable monitoring of both STIs would, furthermore, benefit from strengthening the national reporting system and expanding it to track cases treated in private-sector services, including self-treatment with drugs dispensed by pharmacies.

Our analyses confirm challenges Mongolia faces with STI case reporting; with access to laboratory facilities for diagnosis, which is largely limited to Ulaanbaatar serving just half of the national population; ${ }^{3,7,8}$ and with adherence to syndrome-based case reporting. At present, Mongolia reports negligible numbers of UD cases because most providers do not follow the syndromic approach by which STI patients would get recorded by syndrome whether or not subsequently an etiological diagnosis is established. More consistent implementation of the syndromic approach for both treatment and reporting may improve completeness of treatment (avoiding loss of patients between initial syndromic diagnoses, referral to the laboratory, and waiting time for diagnosis and referral for treatment) as well as case reporting to become more usable for surveillance and planning. Additionally, reporting from pharmacies of clients presenting with UD symptoms might support surveillance. However, self-treatment with over-the-counter drugs should not be encouraged as a treatment policy due to risks of spreading antimicrobial resistance among gonococcal isolates. ${ }^{30}$

In conclusion, model-based estimations based on prevalence surveys suggest that gonorrhoea and chlamydia have declined in Mongolia but remain high. The high STI rates, much of which remains undiagnosed and untreated, bring a substantial burden of sequelae including infertility, pelvic inflammatory disease and ectopic pregnancy; they are a behavioural marker and biomedical cofactor for HIV transmission.

These results, and the wide confidence intervals around most estimates, argue for improved data input though periodic prevalence surveys beyond key populations. Our findings also highlight a largely hidden burden of untreated chlamydia that merits intensification of control efforts beyond routine clinical services. Screening 
should be intensified in primary care settings, among key populations, within antenatal care and for youth, e.g. via school-based clinics. New diagnostic and delivery approaches and affordable point-of-care tests ${ }^{29}$ should facilitate clinic-based and non-clinic-based screening thus improving treatment coverage and surveillance and reducing disease burden.

\section{References}

1. Country Coordinating Mechanism Mongolia. Proposal HIV/AIDS to the Global Fund to fight AIDS, Tuberculosis and Malaria, round 2. UIaanbaatar: Mongolia; 2002 (http://docs.theglobalfund.org/programdocuments/GF_PD_001_f771ef3f-1539-4470-8678-178997acf2bd.pdf).

2. Mongolia Ministry of Health \& HIV/STI program. Mongolian National Strategic Plan on HIV/AIDS/STIs 2010-2015. Ulaanbaatar: Mongolia; 2010 (http://www.wpro.who.int/mongolia/topics/sexually transmitted_infections/nsp_hiv_aids_eng.pdf?ua $=1$ ).

3. Mongolia Ministry of Health \& HIV/STI program. Country Progress Report Mongolia, reporting period 1 January 2012-31 December 2013. Ulaanbaatar: Mongolia; 2014 (http://files.unaids.org/ en/dataanalysis/knowyourresponse/countryprogressreports/2014 countries/MNG narrative report 2014.pdf, accessed 23 October 2017).

4. Mongolia Center for Health Development. Health Statistical Information Annual Reports. Ulaanbaatar: Mongolia; 2016 (http://www. chd.mohs.mn/images/pdf/english\%20indicator-2015.pdf, accessed 23 October 2017).

5. Baigalmaa J, Erdenechimeg C, Narantuya J, Bulbul A, Tugsjargal J, Dolgion $E$, et al. Increasing syphilis notifications in Mongolia: results from national surveillance for 2001-2011. West Pac Surveill Response. 201212 21;3(4):86-93. pmid:23908947 doi:10.5365/ wpsar.2012.3.2.008

6. Purevdawa E, Moon TD, Baigalmaa C, Davaajav K, Smith $\mathrm{ML}$, Vermund $\mathrm{SH}$. Rise in sexually transmitted diseases during democratization and economic crisis in Mongolia. Int J STD AIDS. 1997 Jun;8(6):398-401. pmid:9179652 doi:10.1258/0956462971920190

7. Mongolia National Center for Communicable Diseases AIDS/STI surveillance and research department. Situation of Sexually Transmitted Infections in Mongolia, 2001-2010. Mongolia: Ulaanbaatar; 2011.

8. World Health Organization. Review of the surveillance system for sexually transmitted infections in Mongolia. Mongolia: Ulaanbaatar; 2014 July.

9. Korenromp EL, Mahiané G, Rowley J, Nagelkerke N, Abu-Raddad L, Ndowa F, El-Kettani A, El-Rhilani H, Mayaud P, Chico RM, Pretorius $\mathrm{C}$, Hecht $\mathrm{K}, \mathrm{Wi}$ T. Estimating prevalence trends in adult gonorrhoea and syphilis prevalence in low- and middle-income countries with the Spectrum-STI model: results for Zimbabwe and Morocco from 1995 to 2016. Sex Transm Infect. 2017 March 21;sextrans-2016-052953. pmid:28325771 doi:10.1136/sextrans-2016-052953

10. Newman L, Rowley J, Vander Hoorn S, Wijesooriya NS, Unemo $\mathrm{M}$, Low $\mathrm{N}$, et al. Global estimates of the prevalence and incidence of four curable sexually transmitted infections in 2012 Based on Systematic Review and Global Reporting. PLoS One. 201512 08;10(12):e0143304. pmid:26646541 doi:10.1371/journal. pone. 0143304

11. Chico RM, Mayaud P, Ariti C, Mabey D, Ronsmans C, Chan- dramohan D. Prevalence of malaria and sexually transmitted and reproductive tract infections in pregnancy in sub-Saharan Africa: a systematic review. JAMA. 2012 May 16;307(19):2079-86. pmid:22665107 doi:10.1001/jama.2012.3428

12. Orroth KK, Korenromp EL, White RG, Changalucha J, de Vlas SJ, Gray $\mathrm{RH}$, et al. Comparison of STD prevalences in the Mwanza, Rakai, and Masaka trial populations: the role of selection bias and diagnostic errors. Sex Transm Infect. 2003 Apr;79(2):98-105. pmid:12690128 doi:10.1136/sti.79.2.98

13. El-Kettani A, Mahiané G, Bennani A, Abu-Raddad L, Smolak A, Rowley J, et al. Trends in adult chlamydia and gonorrhea prevalence, incidence and urethral discharge case reporting in Morocco over 1995 to 2015 - estimates using the Spectrum-Sexually Transmitted Infection model. Sex Transm Dis. 2017 Sep;44(9):557-64. pmid:28806354 doi:10.1097/OLQ.0000000000000647

14. National Statistics Office of Mongolia, UNICEF, United Nations Population Fund (UNFPA). Multiple Indicator Cluster Survey in Mongolia, 2013-2014. Report. Mongolia: UNICEF2014 (http:// mics.unicef.org/files?job=W1siZilsljlwMTYvMDMvMzAvMTkvMjkv MDgvODI3LO1vbmdvbGIhXzIwMTNfMTRfTUIDU19FbmdsaXNoL nBkZiJdXQ\&sha=71927f28ca7b8642, https://mics-surveys-prod. s3.amazonaws.com/MICS5/East\%20Asia\%20and\%20the\%20Pacific/Mongolia/2013-2014/Final/Mongolia\%202013-14\%20MICSSISS_English.pdf).

15. Schwebke JR, Aira $T$, Jordan N, Jolly PE, Vermund $S H$. Sexually transmitted diseases in Ulaanbaatar, Mongolia. Int J STD AIDS. 1998 Jun;9(6):354-8. pmid:9671251 doi:10.1258/0956462981922269

16. Binderya G, editor. Etiology of urethral discharge in Ulaanbaatar. Ulaanbaatar: Tackling Infectious Diseases, Information for Action; 2016.

17. Amindavaa $\mathrm{O}$, Kristensen $\mathrm{S}$, Pak $\mathrm{CY}$, Khalzan D, Chultemsuren $\mathrm{B}$, Randall AS, et al. Sexually transmitted infections among pregnant women attending antenatal clinics in Mongolia: potential impact on the Mongolian HIV epidemic. Int J STD AIDS. 2005 Feb;16(2):1537. pmid:15807944 doi:10.1258/0956462053057693

18. Gantumur T, Solongo A, Iliza A, Khandsuren B, Ider D, Narantsetseg V. Report of the STI epidemiological study among pregnant women attending ANC-2008. Ulaanbaatar; 2008.

19. Korenromp EL, Sudaryo MK, de Vlas SJ, Gray RH, Sewankambo NK, Serwadda D, et al. What proportion of episodes of gonorrhoea and chlamydia becomes symptomatic? Int J STD AIDS. 2002 Feb;13(2):91-101. pmid:11839163 doi:10.1258/0956462021924712

20. Erdenetungalag E, Korenromp EL, Badrakh J, Zayasaikhan S, Baya P, Orgiokhuu E, Jadambaa N, Munkhbaatar S, Khishigjargal D, Khad N, Mahiané G, Davaalkham J, Taylor M. Estimating adult female syphilis prevalence, Congenital Syphilis case incidence and adverse birth outcomes due to Congenital Syphilis using the Spectrum Sexually Transmitted Infection surveillance tool, Mongolia 2000-2016. Submitted for publication (September 2017).

21. Tugsdelger S, Amgalan T, Baasansuren A, Erdenetuya G, Ganbold $N$, Jugderjav $B$, et al. Second generation HIV surveillance: Mongolia. Mongolia, Ulaanbaatar: Ministry of Health of Mongolia, World Health Organization, Global Fund supported project on AIDS and TB; 2005 (http://www2.wpro.who.int/NR/rdonlyres/654921DC-89AA40C6-92D3-C97EA1C8AFC4/0/FinalSGSS2005.pdf).

22. Baigalmaa C, Byambaa C. Second Generation HIV/STI Surveillance report, 2002 Ulaanbaatar 2002.

23. Mongolia Ministry of Health, Mongolia Global Fund Supported Project on AIDS and TB. Second Generation HIV/STI Surveillance Report, 2009. Mongolia: Ulaanbatar; 2009 (http://www.aids.mn/files/docs/ sudalgaanii\%20tailan2009.pdf, accessed 23 October 2017). 
24. Mongolia National Center for Communicable Diseases AIDS/ST surveillance and research department. Annual Reports of HIV AIDS, STI Cabinet Registration. Ulaanbaatar: 2017.

25. National Center for Communicable Diseases, Mongolia Ministry of Health. National Guideline on HIV/AIDS and STI Diagnosis and Treatment Care services. approved by health ministerial order \#429. Ulaanbaatar: 2009 \& amended in 2014 by the health ministerial order \#278 (http://www.mohs.mn/uploads/files/4a760aed1 f3a200a96b7b0dd9a6ce07a.pdf)

26. National Statistics Office of Mongolia, UNICEF, United Nations Population Fund (UNFPA). Multiple Indicator Cluster Survey in Mongolia 2010 (MICS-4)Report. Mongolia: UNICEF2013 (https://mics-surveys-prod.s3.amazonaws.com/MICS4/East\%20Asia\%20and\%20 the\%20Pacific/Mongolia/2010/Final/Mongolia\%202010\%20 MICS_English.pdf).

27. Bulbul A, Erdenetungalag E, Davaalkham J, editors. Mongolia HIV estimation using the Spectrum AIDS Incidence Model. 1st National
Conference of Female Scientists: "Tackling Public Health IssuesFemale Scientists' Role in Solution”; 2017 May; Ulaanbaatar: Mongolian Female Scientists' Association.

28. Montana LS, Mishra V, Hong R. Comparison of HIV prevalence estimates from antenatal care surveillance and population-based surveys in sub-Saharan Africa. Sex Transm Infect. 2008 Aug;84 Suppl 1:i78-84. pmid:18647871 doi:10.1136/sti.2008.030106

29. Global health sector strategy on sexually transmitted infections 2016-2021. Towards ending STIs. Report. Report No.: WHO/ RHR/16.09. Geneva: World Health Organization; 2016 June (http:// apps.who.int/iris/bitstream/10665/246296/1/WHO-RHR-16.09eng.pdf?ua=1).

30. Report on global sexually transmitted infection surveillance 2015. Geneva: World Health Organization; 2016 August (http:// www.who.int/reproductivehealth/publications/rtis/stis-surveillance-2015/en/). 